\title{
The theory of functional systems as a basis of the infographic modelling anthropotechnics
}

\author{
Vitaly Chulkov ${ }^{1, *}$ \\ ${ }^{1}$ Moscow State University of Civil Engineering, 26, Yaroslavskoye Shosse, 129337, Moscow, Russia
}

\begin{abstract}
The need for the functioning of the complex system "man technology - environment, MTE" predetermined the study and development of mechanisms that allow a person to remain "almost healthy" under the influence of various pathogenic factors of technology and the environment, to bear a variety of stress and activity loads. A characteristic feature of the studies in this area, carried out in our country at the end of the 20th and beginning of the 21 st centuries, is a pronounced social orientation, focus on the priority of human interests in the triad of components of the MTE system, and indispensable reliance on the logical and philosophical modelling of the object of study.
\end{abstract}

\section{Introduction}

Studies of the MTE system, initiated in various fields of science and activity (in philosophy, healthcare, labor protection and safety, ecology, etc.), have covered technical sciences as well as borderline (marginal) areas of interdisciplinary research (for example, medical technology).

A new level of research is associated with the development and application of a systematic approach to understanding the interconnections of the individual components of the MTE system, with the study of physiological processes in this system, with the formation of the necessary level of life safety in it. These studies require logical thinking, as an indispensable tool for cognition, since the formulation of a scientific hypothesis, the purpose of the study, and the tasks realized to achieve it is often more difficult and important than the research itself.

\section{Materials and methods}

Logical thinking requires to accurately and unambiguously sum up thoughts, to be able to display the result of the study of the MTE system by means of infographic modeling: one-, two-, three- and more parameter dependencies; plain and spatial geometric images of the theory of multipoint logics; figures and tables; charts and histograms; other sign systems $[1]$.

\footnotetext{
*Corresponding author: aljurgaitis@gmail.com
} 
The MTE system is human-machine or natural-artificial. The presence of a person in it, as the most important component, determines a number of qualitatively new properties [2]:

"compliance" of a person to the spatio-temporal continuum of the surrounding world (internal and external environment in relation to the MTE system);

adaptability to episodic and periodic environmental events;

selectivity of protection from the environment and communication with it;

learning ability, as the formation of the same type of reaction to the same type of situation;

an advance reflection of reality, the idea of which was discovered by I.P. Pavlov and developed by P.K. Anokhin [3 et al.] and K.V. Sudakov [4 et al.].

Physiological cybernetics, as applied to the life of biological systems, has identified the principle of self-regulation: a form of life activity in which a deviation of a function from the level that ensures normal life is the cause of the return of this function to its original level.

Theory of functional systems, which was formulated in 1935 by P.K. Anokhin, considers such systems morphofunctional units of self-regulation. Twelve years earlier than Norbert Wiener, the student of I.P. Pavlov, academician P.K. Anokhin, formulated, experimentally identified and proved by experiments on animals the concept of "reafference", which is essentially identical to the Wiener concept of "feedback".

Functional systems are self-organizing and self-regulating dynamic organizational structures, the activity of all components of which contributes to the achievement of adaptive results useful for the object of study. These may be the results of the behavioral, social, or mental activities of an individual, a group of people, or a population. Functional systems may initially be characteristic of the object of study (for example, the MTE system) or, if necessary, may arise during the operation or reconstruction of the MTE system.

Having perfected the basic infographic model of a functional system (Fig. 1), P.K. Anokhin revealed a sequence of its nodal stages that have specific qualities that are characteristic only of a functional system. The student of P.K. Anokhin, academician K.V. Sudakov, within the framework of the theory of functional systems, introduced the term and concept of "systematic quantum of behavior" [5, 6, etc.], investigated its basic model, and revealed the varieties of system quantization of life.

V.O. Chulkov investigated the concept of "systematic quantum of activity" [7, 8, etc.] in the field of anthropotechnics of infographic modeling of MTE systems.

V. M. Lebedev adapted the concept of systematic quanta to the field of construction production, design and scheduling of construction processes and objects $[9,10$, etc.]. Such a functional and systematic approach to the development and implementation of in-line construction projects using systematic quanta of construction processes and objects based on infographic models is very convenient and relevant. Images of informational guiding vectors entwined with ascending quanta of material, technical and energy resources in the space-time continuum for different hierarchical levels of control are used in anthropotechnical management. 


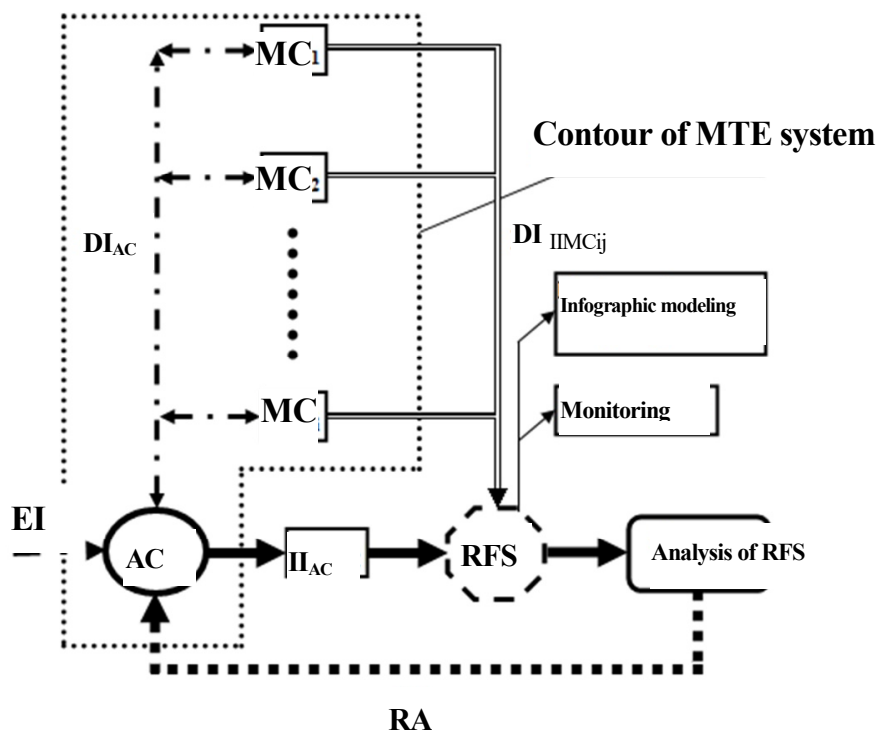

Fig. 1. Basic infographic model of the MTE functional system (2003, [4]). Where : FS - functional system; EI - external impact on FS; AC - accented component of MTE FS; $\mathrm{MC}_{\mathrm{ij}}$ - marginal (peripheral) components of MTE FS; $\mathrm{DI}_{\mathrm{AC}}$ - direct impact (regulation) by AC in order to achieve the result of FS; $\mathrm{II}_{\mathrm{MCij}}$ indirect impact (regulation) by $\mathrm{MC}_{\mathrm{ij}}$ in order to achieve the result of $\mathrm{FS}$; $\mathrm{II}_{\mathrm{AC}}$ - indirect impact (regulation) of $\mathrm{AC}$ on $\mathrm{MC}_{\mathrm{ij}}$ in order to achieve the result of FS; RFS - the result of the FS; RA - reafference (connection).

Scientific research and the results of the school of P.K. Anokhin formed the basis for the formation of new scientific areas: physiological cybernetics, neurocybernetics, functional neurochemistry, the theory of integrative activity of a neuron, anthropotechnical management, etc.

The significance of the ideas of the school of thought of P.K. Anokhin - K.V. Sudakov is to a large extent determined by the prospects that they open up for new studies and studies of functional systems in various scientific and practical applications.

The emphasis on one of the components of the MTE functional system (for example, on the "man" component) transfers the remaining components of this system to the category of marginal with respect to the accented one. A two-way relationship is established between $\mathrm{AC}$ and MCij, while there is only one-sided impact (regulation) on RFS.

A change in the result of the RFS functional system (Fig. 1) is revealed in the process of its infographic modeling (visual display of the result), monitoring (constant or periodic monitoring and instrumental engineering diagnostics) and analysis of the state of a useful adaptive result, on compliance of the current state of which reafference (feedback) immediately notifies the functional system. Under the impact of reafference, mechanisms and technologies ensuring the restoration of the result required for the metabolism of the human body or the normal course of its social and labor activity are involved in the functional system selectively and purposefully (teleologically).

Reafference is the basis for assessing the stages of behavior aimed at achieving the intended result. It determines the processes of self-regulation of a functional system [2].

The central element of the infographic model (Fig. 1) of any functional system is an adaptable result useful for the object of study. Deviation from this result as from the nominal value of the specific characteristics of the object of study, ensuring the normal level of life safety, selectively mobilizes the mechanisms and technologies for 
compensation of pathogenic effects appropriate to the task of restoring the desired level of result through the feedback channel (reafference) [11].

Monitoring provides continuous control of the level and quality of life safety, and infographic modeling allows performing procedures for determining and regulating the quality of life safety through the visual channel, which supplies the human brain with up to $80 \%$ of all processed information.

The aforementioned characterizes the cybernetic properties of functional systems: the dynamics of its work, regulation by the final effect, and information exchange. The modern level of scientific knowledge allows, paying tribute to the discoverers ("reafference" by P.K. Anokhin in physiological cybernetics, 1935; "feedback" by N. Wiener in technical devices, 1948), using these synonymous concepts in the study of complex systems, an example of which is the MTE system.

Important features of the functional systems:

- isomorphism of their particular infographic models (the presence in the models of a common "invariant" structural component and specific structural components inherent to only a particular functional system);

- holographic principle of organizing the components of a functional system (each element of a functional system reflects in its properties the activity of the system as a whole and, most importantly, reflects the current state of its useful adaptive result); therefore, the fundamental approach of construction anthropotechnics is justified;

- selective involvement of elements in functional systems (the same elements can be used in different functional systems to achieve different adaptive results; or otherwise, compensation for the same adaptive result, i.e. bringing it to its nominal state or to lafe safety norms in the MTE system, can be implemented using different elements of the functional system; such interchangeability of elements can be pre-designed or emergency, off-nominal); selective involvement of elements can be carried out both in the operational (requiring speed of actions) and strategic (steady) modes);

- interaction of elements in functional systems (an individual element is not included in the activity of the functional system passively, but actively interacts with other selectively involved elements, which contributes to the achievement of a useful adaptive result of the functional system);

- dominant hierarchy of functional systems of one object of study (to achieve the quality of an adaptive result by the object of study (for example, the MTE system) at any given time, one functional system is most important (dominates); the dominance of systems is determined by their biological (for individual) or social (for a collective) significance); this characteristic of functional systems was discovered by A.A. Ukhtomsky;

- sequential interaction of several functional systems related to one object of study (the activity of one functional system in time replaces the activity of another functional system, which once again confirms the dominance in the hierarchy of functional systems of the object of study).

The variety of vital functions in the MTE system considered in the dynamics allows distinguishing a sequential series of effective segments. K.V. Sudakov suggested that each such productive interval of the vital activity of the object of study, determined by a special functional system, should be considered as a "systematic quantum" or "systemoquantum" $[2]$.

"Systemoquants" are found in various objects of study and functional systems that serve this object. They apply to the behavior of the elements of the studied MTE systems, when each "systemoquantum" of behavior includes phasr and final behavioral results satisfying the adaptive result.

"Systemoquants" of purposeful activity are formed on the basis of different needs: biological in humans or social in the collective (Fig. 2). 


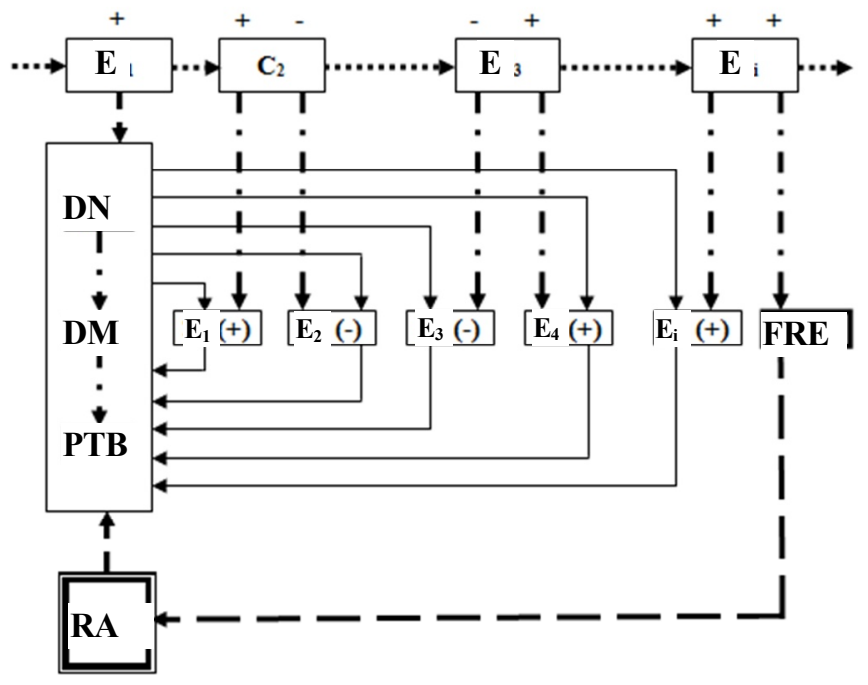

Fig. 2. Basic infographic model of "systemoquantum" according to K.V. Sudakov (2003, [4]). Where: $E_{1}, E_{2}, \ldots, E_{i}$ - events in the external environment (with respect to the considered system quantum); DN dominant need; DM - dominant motivation; PTB - program of targeted behavior; $\mathrm{P}_{1}, \mathrm{P}_{2}, \ldots, \mathrm{P}_{\mathrm{j}}$ - phase (intermediate) positive $(+)$ and negative (-) results of behavior; FRE - the final result of the event, including the result of the "one pass" of the functioning algorithm of the "systemoquantum" to satisfy the DN; RA reafference ("reinforcement" according to K.V. Sudakov).

\section{Results and discussion}

The basic infographic model of "systemoquantum" reflects the emergence of a dominant need, the formation on its basis of a dominant motivation and a program of targeted behavior that ensures the achievement of phase $(\mathrm{P} 1, \mathrm{P} 2, \ldots, \mathrm{Pj})$ and final (FRE) results that satisfy the dominant need .

"Systemoquants" are distinguished by the nature and quality of both the dominant needs and the processes (technologies) of their satisfaction. Infographic models of "systemoquants" have isomorphic (as defined by P.K. Anokhin, [12]) architectonics.

For the MTE system (for example, for the totality of processes of construction production during the erection of buildings and structures), as a complex object of study in construction anthropotechnics, the leading system-forming factor of activity is a useful adaptive result. In the production activity of a particular person working on the construction site, such a result has not only individual mercantile aspects, but also a certain social significance, since in order to achieve general socially significant results, this person is forced to mobilize his physiological resources (labor input).

The sequential interaction of different functional systems in relation to the dynamics of the formation of the object of study (MTE system) is called the systemogenesis of behavioral acts, SBA.

SBA processes are widespread by the school of thought of P.K. Anokhin for almost the entire period of a person's individual life. They are especially interesting when studying the laws of the formation of systemic relationships in human populations when performing various types of social activities, including industrial ones. This type of SBA is called population systemogenesis, PS. 


\section{Conclusion}

In the MTE system, information, as a measure of the relationship between a person, technology and the environment in their various forms, forms a single sphere of ideal phenomena of nature and society. In 1996, I.I. Yuzvishin put forward a hypothesis that the organizational structure of the information space (information field) is a cellular model and is an entity that is characterized by wave, resonance, and oscillatory processes [13].

According to the informational-cellular model of I.I. Yuvshin, information transmission is carried out not by elementary particles (atoms, electrons or molecules) that propagate in the material space, but by resonant relations between waves.

Other researchers express and confirm similar hypotheses by their reasoning and statistical materials of long-term experiments $[14,15,16]$.

\section{References}

1. V.O. Chulkov, Infographics. Course of lectures (Moscow State University of Civil Engineering named after V.V. Kuybyshev, Moscow, 1991)

2. K.V. Sudakov, Physiology. Fundamentals and Functional Systems: Lecture Course (Medicine, Moscow, 2000)

3. P.K. Anokhin, Questions of philosophy 7, 97 (1962)

4. P. Oleinik, A. Yurgaytis, MATEC Web of Conferences 117, 00130 (2017) https://doi.org/10.1051/matecconf/201711700130

5. K.V. Sudakov, Functional systems (Publishing House of the Russian Academy of Medical Sciences, Moscow, 2011)

6. K.V. Sudakov, I.A. Kuzichev, A.B. Nikolaev, V.I. Shchelkanov, The evolution of terminology and schemes of functional systems in the school of thought of P.K. Anokhin (European printing systems, Moscow, 2010)

7. V.O. Chulkov, Life safety. Organizational and anthropotechnical reliability of functional systems of the mobile environment of construction production. Series "Infographic Fundamentals of Functional Systems" (IFFS) (ASV Publishing House, Moscow, 2003)

8. V.O. Chulkov, Organizational and technological innovations of housing and communal and investment and construction complexes in the development of the city: International collection of scientific papers (Moscow State Academy of Public Utilities and Construction, Moscow, 2012)

9. V.M. Lebedev, System engineering and systemoquants of construction production: monograph (INFRA-M, Moscow, 2018)

10. V.M. Lebedev, Systemoquants of technological processes in the production line method of construction of facilities and complexes: monograph (INFRA-M, 2018)

11. A. Wilson, M. Wilson, Information, computers, and system design (Mir, Moscow, 1968)

12. K.V. Sudakov, Sanatron. The system of assessment and rehabilitation of early violations of the physiological functions of a person in real life conditions (Gorizont, Moscow, 2001)

13. P. Oleinik, A. Yurgaytis, MATEC Web of Conferences 193, 05010 (2018) https://doi.org/10.1051/matecconf/201819305010

14. V.G. Zilov, K.V. Sudakov, O.I. Epstein, Elements of information biology and medicine (Moscow State Forest University, Moscow, 2000) 
15. K.V. Sudakov, V.O. Chulcov, R.R. Kazaryan, O.S. Glazachev, N.V. Dmitrieva, N.M. Komarov, Antropotechnics: Norm in every living thing and artificial beings (SvR-ARGUS, Moscow, 2013)

16. K.V. Sudakov, Functional systems (Publishing House of the Russian Academy of Medical Sciences, Moscow, 2011) 